\title{
Cooperative Citizen Science for Robot-Mediated Telerehabilitation
}

\author{
Roni Barak Ventura ${ }^{1}$, Shinnosuke Nakayama ${ }^{1}$, Preeti Raghavan ${ }^{2}$, Oded Nov ${ }^{3}$, Maurizio Porfiri ${ }^{1,4}$ \\ ${ }^{1}$ Department of Mechanical Engineering, New York University Tandon School of Engineering \\ 6 MetroTech Center, Brooklyn, NY 11201 \\ rbv215@nyu.edu; sn2286@nyu.edu; mporfiri@nyu.edu \\ ${ }^{2}$ Department of Rehabilitation Medicine, New York University School of Medicine \\ 400 East $34^{\text {th }}$ Street, New York, NY 10016 \\ Preeti.raghavan@nyulangone.org \\ ${ }^{3}$ Department of Technology Management and Innovation, New York University Tandon School of Engineering \\ 5 MetroTech Center, Brooklyn, NY 11201 \\ onov@nyu.edu \\ ${ }^{4}$ Department of Biomedical Engineering, New York University Tandon School of Engineering \\ 6 MetroTech Center, Brooklyn, NY 11201 \\ mporfiri@nyu.edu
}

\begin{abstract}
Robot-mediated telerehabilitation has made great strides toward patient-tailored, cost-effective treatments. However, lack of motivation often leads to patients' non-compliance with their physical therapy regimen, undermining the potential advantages of telerehabilitation technologies. Embedding citizen science into robotics-based rehabilitation has been shown to increase patients' motivation to engage in otherwise mundane exercises. Here, we explore the feasibility of bolstering engagement in physical therapy through social interactions in citizen science context. We developed an online citizen science platform in which users work in pairs to classify images collected by an aquatic robot in a polluted water canal in Brooklyn, New York. The classification involves labeling objects that appear in the images and removing irrelevant labels. The application was interfaced by a haptic device for fine motor rehabilitation. We present preliminary results for the levels of engagement of healthy users performing the activity, with and without a cooperating peer.
\end{abstract}

Keywords: citizen science; cooperation; engagement; fine motor; haptic device; motor performance; physical therapy; telerehabilitation.

\section{Introduction}

Recent advances in robotic rehabilitation technologies have enabled the delivery of controlled and customizable physical therapy to patients' homes [1]. Remote administration of physical therapy has the potential to increase the accessibility to high-quality medical care [1], empower therapists to treat a larger number of patients simultaneously [2], and make treatment more affordable [3]. While telerehabilitation is successfully implemented in several instances, low compliance is often observed among patients in carrying out their prescribed physical exercise. Lack of motivation has been identified as a major factor underpinning non-compliance with the physical therapy regimen [4]. In efforts to overcome non-compliance, a large body of research has investigated different motivational interventions, largely focusing on gamification [5]. However, the repertoire of motivational interventions remains underexploited as designers rarely consider the end-users' interest and intellect. Moreover, gamification strategies typically do not attend to the needs of age group of the majority of rehabilitation patients [6][7].

Previously, we have demonstrated that embedding rehabilitation exercise with citizen science can motivate patients to repeat physical exercise [8]. In citizen science, members of the public participate in research led by professional scientists by collecting or analyzing large datasets [9]. Unlike traditional gamification strategies, citizen science employs a wide range of motivational drivers, including intrinsic motives [10]. However, citizen science could benefit from the introduction of social interactions to further enhance contributions to citizen science projects [11], [12]. 
In this study, we sought to bolster engagement in citizen science-based physical therapy by incorporating cooperation among citizen scientists. We hypothesized that cooperative tasks would motivate users to extend their contribution to citizen science projects by increasing the amount of data they process (productivity) and the amount of time they spend performing the task (persistence). Moreover, we expected that the integration of cooperation would impact motor performance, prompting users to exert higher levels of physical effort. To test these hypotheses, we created a cooperative online platform for Brooklyn Atlantis, an environmental citizen science project for monitoring the Gowanus Canal, a highly-polluted canal in Brooklyn, New York [13]. The online application enabled users to analyze pictures of the canal taken by a robotic boat using the Novint Falcon, a haptic gaming controller capable of delivering effective fine motor hand rehabilitation [8], [14]. Through the application, pairs of volunteers were asked to sort a list of keywords that may describe the objects in the images, where one user allocates labels that describe objects in the image, while the other user removes irrelevant labels. Here, we report preliminary results for the engagement of healthy individuals using the platform; detailed evaluation on a larger population will be the object of future publications.

\section{Methods}

\subsection{Hardware and Software}

The cooperative citizen science application was developed using Unity 3D (Unity Technologies, San Francisco, California) and interfaced with the Novint Falcon (Novint Technologies, Albuquerque, New Mexico), a low-cost haptic device whose potential use in rehabilitation has been demonstrated by our group and other researchers [8], [14]. The application displays a $360^{\circ}$ image of the Gowanus Canal on a computer screen (Fig. 1). The image can be explored in spherical coordinates whereby moving the end effector along the $\mathrm{x}$-axis (left-right) translates into azimuthal rotation and moving of the end-effector along the y-axis (up-down) translates into elevational rotation. Since zoom is not offered and motion along the z-axis (push-pull) is not functional, a highly resistive force is applied in this direction to prevent pushing and pulling of the end-effector. Deviation from the z-axis is conveyed to users through a black circle and a radiating cone, portraying where the user is facing and how far off the axis they are located (Fig. 1). In addition, a visual feedback is added to the application, such that the image of the Gowanus Canal will fade in response to motion along the z-axis. When two users are paired, each has independent control over exploration of the $360^{\circ}$ image.

Two tasks are to be completed in this image classification project. One task consists of tagging objects in the image (e.g. factory, crane, or trash), while the other consists of eliminating labels that are not found in the image. Once assigned, labels are replaced by others from a predetermined sequence of 49 labels, created by citizen scientists in previous Brooklyn Atlantis projects [8], [12]. Cooperating users are able to view their partner's actions in real time as both users can see the labels assigned to the image as well as a list of the eliminated labels forming below the trash bin (Fig. 1). Social presence is further conveyed by highlighting labels in red, indicating that the user's partner has selected them.

To avoid over-saturation of images with tags and to maintain succession of the image classification process, every image is replaced by another image after five tags were assigned. A tag counter is displayed on the screen to inform the user of the number of tags left to be assigned to the current image (Fig. 1). Finally, users are able to withdraw from the activity by pressing the red "Quit" button on the screen (Fig. 1). 


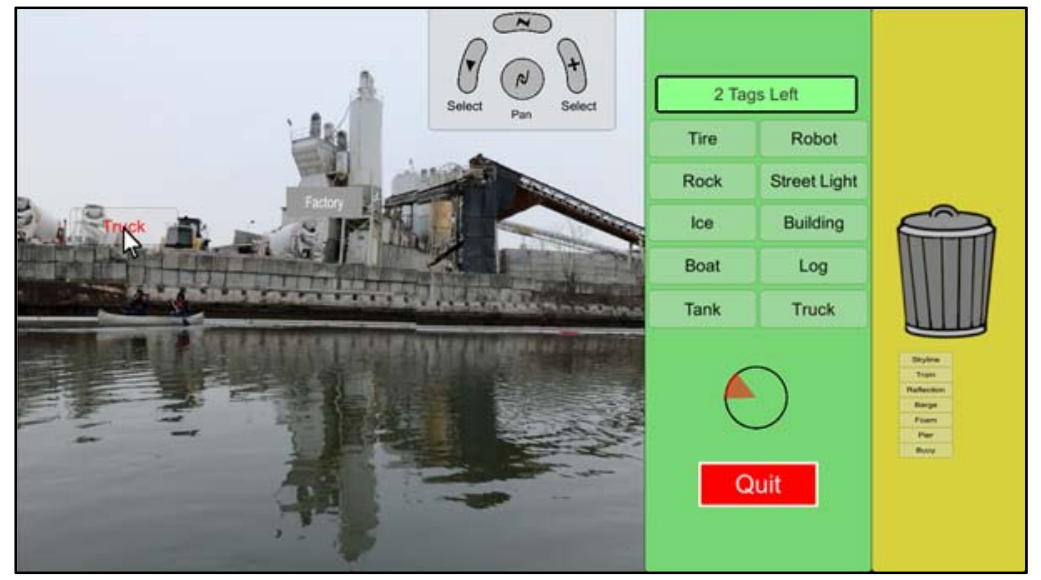

Fig. 1: A screenshot of the user interface. The user is allocating the label "Truck" to the $360^{\circ}$ image on the left, after having already allocated a tag containing the word "Factory". A counter with the number of labels left to be tagged in the current image is displayed on the top of the green panel. Below it, there is a list of 10 labels and a visual feedback for deviation from the z-axis. A Quit button is situated at the bottom of the green panel. In the yellow panel, there is a garbage bin for eliminating labels that do not describe objects in the current image. The seven labels below it were eliminated by the user.

\subsection{Experimental Procedure}

This study was approved by the institutional review board (IRB) at New York University (IRB\#-FY2016-184). Thus far, 30 members of the university community were recruited and subjected to one of two conditions: cooperation (20 volunteers) and control (10 volunteers). Cooperating subjects carried out the activity in pairs such that one person performed only tagging and the other performed only trashing. Control subjects performed both tagging and trashing tasks, alone (Fig. 2).

Volunteers were recruited individually and brought into a private room. To simulate online cooperation as envisioned in robotics-based telerehabilitation, cooperating volunteers were brought into two separate private rooms and paired online, not knowing each other. Before beginning the activity, participants were given an overview of Brooklyn Atlantis, followed by a tutorial teaching them how to use the Novint Falcon and the application. Cooperating participants were made aware that they will be working together with a partner and instructed to complete their assigned task only. Upon signing a consent form, participants began carrying out their tasks. They performed the exercise until they pressed the quit button. All participants were able to withdraw from the activity at any time.

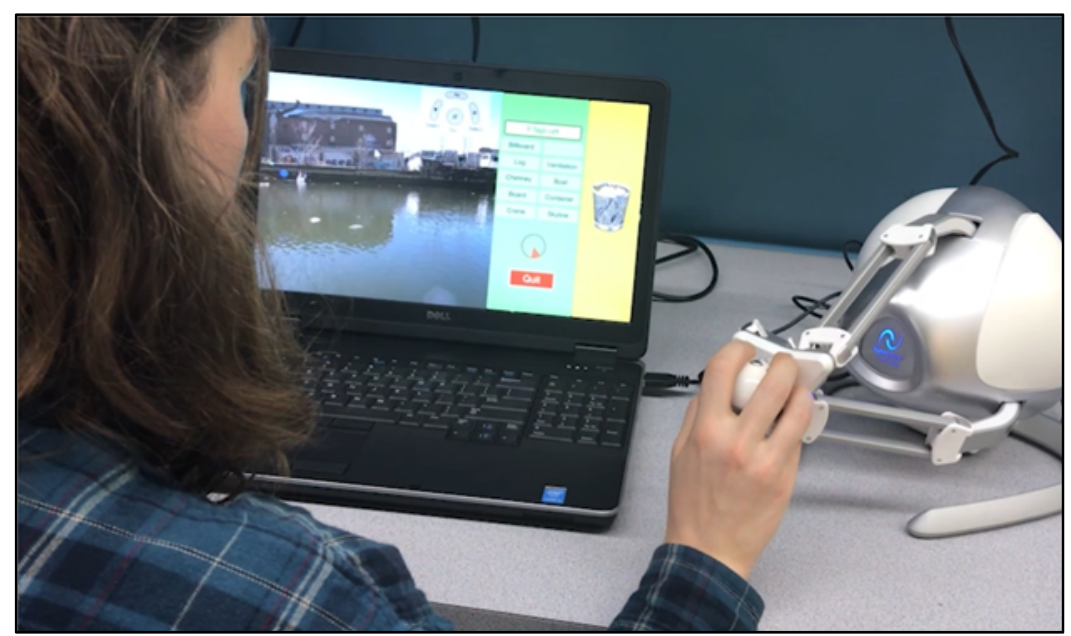

Fig. 2: An illustration of a control user performing the citizen science activity using the Novint Falcon. 


\subsection{Data Collection and Analysis}

For each user, information on label allocation (namely identity of user performing the allocation, content of the label and the time its allocation) was collected to evaluate their engagement, operationalized as persistence and productivity. Persistence was measured as the amount of time spent performing the activity until withdrawal and productivity was measured as the number of labels (tagged or trashed) [15].

To evaluate users' motor performance, the position of the Novint Falcon end-effector in 3D space was recorded at a sampling rate of 60 positions per second. Two motion metrics were evaluated from the end-effector's trajectory: mean speed and peak speed (Fig. 3). The instantaneous speed was estimated using a backward Euler scheme on the sampled positions. The mean speed was computed by averaging instantaneous values over the whole trajectory and the peak speed as the 90th percentile from the trajectory (Fig 3.) [16].

Each of the four variables was fitted into a generalized linear-mixed effects model [17], specifying condition (cooperation or control) as an independent variable and pair identity as random effects (using R 'lme4' package version $1.1-15$; [18]). To improve the normality of the model residual, a gamma family with a log link was specified. The significance of the influence of conditions was tested using a likelihood ratio test, comparing the model against a null model in the absence of the condition as the independent variable. Post-hoc analysis was performed using Dunnett's test when a significant effect was found (using R 'multcomp' package version 1.4-8; [19]).

For all statistical tests, the level of significance was set to $\alpha=0.05$.

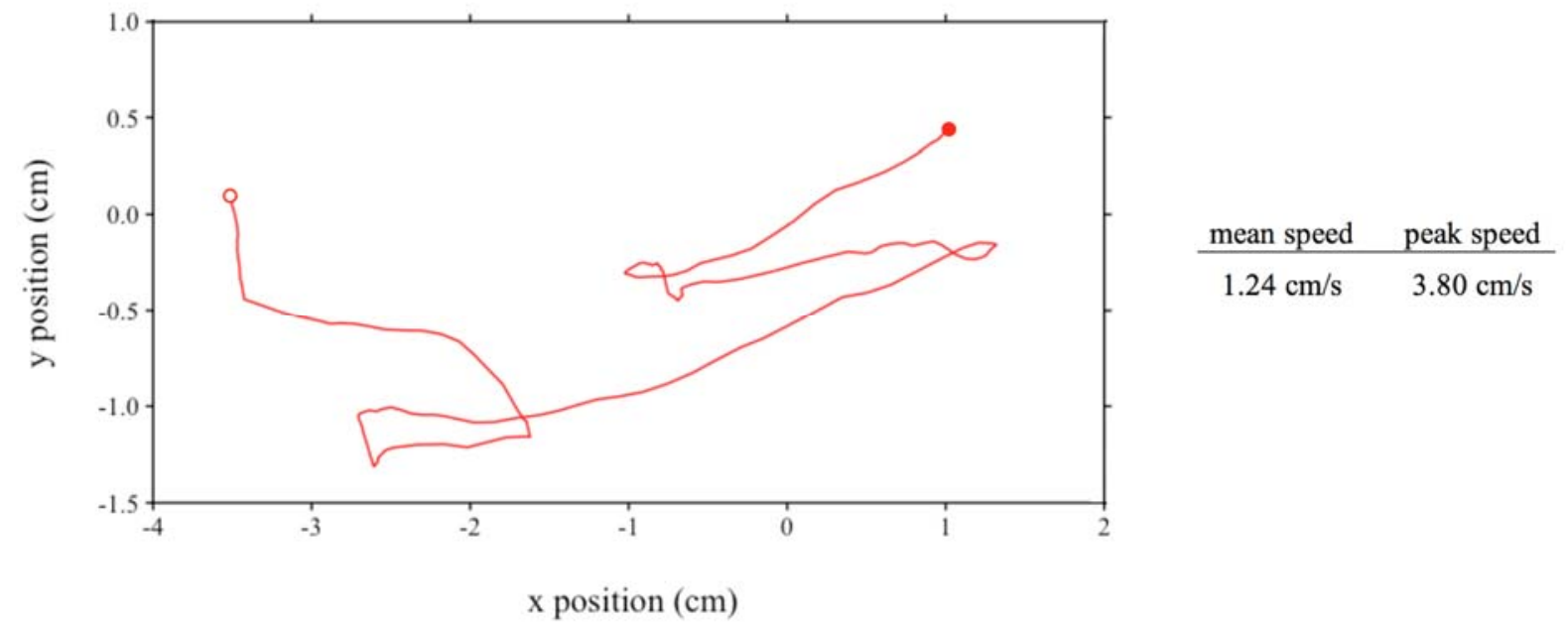

Fig. 3: An example of end-effector trajectory data captured during 10 seconds of a trial, starting from the filled circle and ending at the empty circle. Mean speed and peak speed represent performance within this time segment.

\section{Results}

On average, users spent $15.36 \pm 1.09$ minutes (mean \pm standard error), processing $45.06 \pm 5.93$ labels (Fig. 4). Neither persistence nor productivity were found to differ between cooperating and control users $\left(\chi_{1}^{2}=2.383, p=0.122\right.$ and $\chi_{1}^{2}=1.705$, $\mathrm{p}=0.191$, respectively).

With respect to motor performance, while no difference was registered in mean speed $\left(\chi_{1}^{2}=1.610, p=0.204\right)$, cooperating users reached a significantly higher peak speed relative to control users $\left(\chi_{1}^{2}=5.549, \mathrm{p}=0.018\right.$; Fig. 4). 
A)

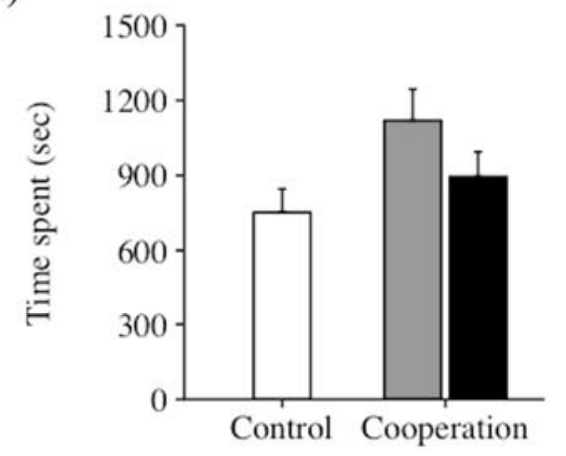

C)

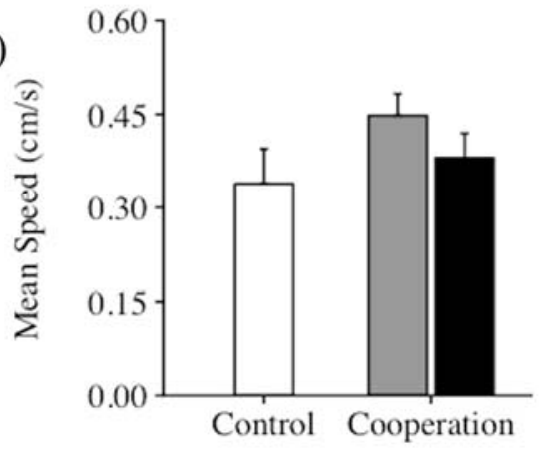

B)

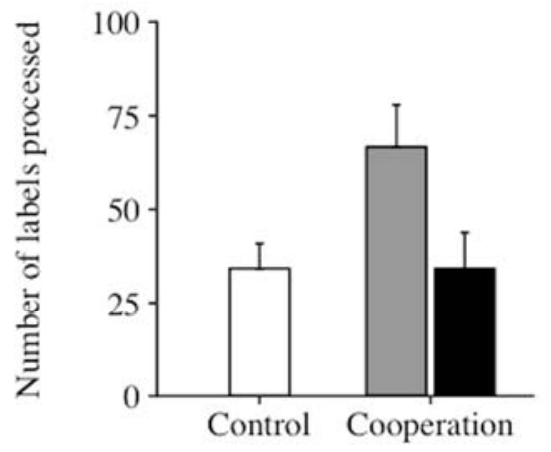

D)

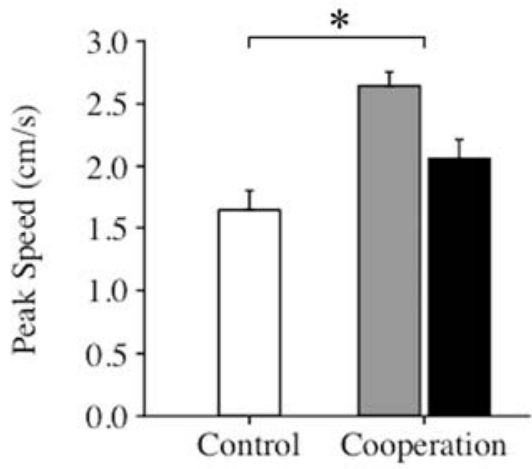

Tag

Trash

Fig. 4: The influence of cooperation on A) persistence, B) productivity, C) mean speed, and D) peak speed. The vertical lines represent standard errors. * represents statistically different means among conditions.

\section{Conclusion}

Citizen science is an effective means for increasing patients' motivation to engage in rehabilitation treatments [8], thereby potentially providing an effective means for improving rehabilitation outcomes. In an effort to further enhance engagement in rehabilitation exercise by tapping into human social motives, we developed a cooperative online citizen science platform and initiated a series of experiments to study the influence of cooperation on engagement in healthy users.

Thus far, our results suggest that the integration of cooperation into citizen science-based rehabilitation exercise does not necessarily promote engagement but it may impact motor performance. Since citizen science projects are essentially social projects relying on collaborative efforts from a multitude of individuals, and inherently employ a wide array of motives to attract and retain volunteers [10], it is tenable that motivational interventions such as cooperation offer only a weak enhancement to engagement. Nonetheless, enabling a direct interaction between volunteers during citizen science tasks opens the door toward novel, creative motivational interventions. For example, an online connection could enable users to send each other encouraging messages while performing the activity. Strengthening social interactions through similar interventions could increase engagement as well as alleviate the loneliness many patients with disability experience [7].

By inspection of the graphs in figure, we notice that task assignment can impact both engagement and motor performance. Specifically, users assigned with tagging seemed more engaged in the activity than those who were assigned with trashing. It is plausible that the unequal division of labor, such that each participant carries out a different task, has associated each task with a different level of identifiability, whereby the contribution of taggers is more recognized and valued by the group [20]. As a result, taggers were more motivated to contribute to the project and exhibited higher levels of engagement. This notion calls for an additional study to explore how engagement is influenced by collaboration, such that 
users are assigned with an identical task, rather than cooperation. Perhaps, the notion of collaboration itself could be tailored by modulating the experimental procedure, thereby strengthening or weakening the degree of interdependence between users.

We presented preliminary evidence for the utility of cooperation in enhancing engagement in citizen science-based telerehabilitation. We observed that task assignment within cooperation can modulate user engagement and motor performance. The value of this finding can be relayed to other domains that build on user participation and engagement, including online social platforms, crowdsourcing efforts, and general game design. Ultimately, we anticipate citizen science-based telerehabilitation will become ubiquitous and help patients reach their full potential recovery.

\section{Acknowledgements}

This study was supported by the National Science Foundation under award number CBET-1604355. RBV would like to acknowledge the Mitsui USA Foundation for their generous support. All the authors would like to thank M. Nadini for helping conduct experiments, and S. Richmond and T. J. Tolbert for software development.

\section{References}

[1] C. O. Cherry et al., "Expanding stroke telerehabilitation services to rural veterans: a qualitative study on patient experiences using the robotic stroke therapy delivery and monitoring system program," Disabil. Rehabil. Assist. Technol., vol. 12, no. 1, pp. 21-27, 2017.

[2] A. Song, C. Wu, D. Ni, H. Li, and H. Qin, "One-Therapist to Three-Patient Telerehabilitation Robot System for the Upper Limb after Stroke,” Int. J. Soc. Robot., vol. 8, pp. 319-329, 2016.

[3] J. C. Perry, J. A. Ruiz-Ruano, and T. Keller, "Telerehabilitation: Toward a cost-efficient platform for post-stroke neurorehabilitation," in IEEE International Conference on Rehabilitation Robotics, 2011, pp. 1-6.

[4] R. K. Dishman and W. Ickes, "Self-motivation and adherence to therapeutic exercise," J. Behav. Med., vol. 4, no. 4, pp. 421-438, 1981.

[5] J. W. Burke, M. D. J. McNeill, D. K. Charles, P. J. Morrow, J. H. Crosbie, and S. M. McDonough, “Optimising engagement for stroke rehabilitation using serious games," Vis. Comput., vol. 25, no. 12, pp. 1085-1099, 2009.

[6] E. Flores, G. Tobon, E. Cavallaro, F. I. Cavallaro, J. C. Perry, and T. Keller, "Improving patient motivation in game development for motor deficit rehabilitation," in Proceedings of the 2008 International Conference in Advances on Computer Entertainment Technology - ACE '08, 2008, p. 381.

[7] W. Ijsselsteijn, H. H. Nap, Y. de Kort, and K. Poels, "Digital game design for elderly users," in 2007 Conference on Future Play, 2007, pp. 17-22.

[8] J. Laut, F. Cappa, O. Nov, and M. Porfiri, "Increasing patient engagement in rehabilitation exercises using computer-based citizen science," PLoS One, vol. 10, no. 3, p. e0117013, 2015.

[9] E. Hand, "Citizen science: People power," Nature, vol. 466, no. 7307, pp. 685-687, 2010.

[10] O. Nov, O. Arazy, and D. Anderson, "Dusting for science: motivation and participation of digital citizen science volunteers," in Proceedings of the 2011 iConference, 2011, pp. 68-74.

[11] S. Nakayama, T. J. Tolbert, O. Nov, and M. Porfiri, "Social information as a means to enhance engagement in citizen science-based telerehabilitation," J. Assoc. Inf. Sci. Technol.

[12] J. Laut, F. Cappa, O. Nov, and M. Porfiri, "Increasing citizen science contribution using a virtual peer," J. Assoc. Inf. Sci. Technol., vol. 68, no. 3, pp. 583-593, 2017.

[13] J. Laut, M. Porfiri, and O. Nov, "Brooklyn Atlantis: A robotic platform for environmental monitoring with community participation," in Proceedings of the 1st International Conference of Control, Dynamic Systems and Robotics, May 15-16, 2014.

[14] B. Lange, S. Flynn, and A. Rizzo, "Initial usability assessment of off-the-shelf video game consoles for clinical game-based motor rehabilitation," Phys. Ther. Rev., vol. 14, no. 5, pp. 355-363, 2009.

[15] M. Touré-Tillery and A. Fishbach, "How to measure motivation: A guide for the experimental social psychologist," Soc. Personal. Psychol. Compass, vol. 8, no. 7, pp. 328-341, 2014. 
[16] K. Kaushik, E. Sharifi, and S. E. Young, "Computing Performance Measures with National Performance Management Research Data Set," Transp. Res. Rec. J. Transp. Res. Board, vol. 2529, no. 2529, pp. 10-26, 2015.

[17] A. F. Zuur, E. N. Ieno, N. J. Walker, A. A. Saveliev, and G. M. Smith, Mixed effects models and extensions in ecology with R. Springer, New York, NY, 2009.

[18] D. Bates, M. Mächler, B. Bolker, and S. Walker, "Fitting linear mixed-effects models using lme4," J. Stat. Softw., vol. 67 , no. 1, pp. 1-48, 2015.

[19] F. Bretz, P. Westfall, and T. Hothorn, Multiple comparisons using R. Boca Raton, Florida, USA: Chapman and Hall/CRC, 2010.

[20] D. M. Shaw, "Size of Share in Task and Motivation in Work Groups," Sociometry, vol. 1960, no. 2, pp. 203-208, 1960. 